\title{
Prediction of Sensory Attributes of Fresh Beef Using Advanced Spectral Analysis
}

\author{
Noha Morsy \\ Department of Food Science and Technology (Home Economics Branch), Faculty of Agriculture, Suez Canal \\ University, Ismailia, Egypt
}

Received: $14 / 2 / 2020$

\begin{abstract}
One of the main roles of home economics tasks is to seek high quality food with desirable palatability and lowest costs. The development of an advanced method to guarantee the sensory and nutritional parameters becomes very crucial in modern food technology. Spectral imaging technology has more advantages over the other conventional methods in food quality evaluation due to rich information it provides. The main aim of the current study was to present a method for non-invasive determination of sensory quality attributes of beef based on spectral analyses of spectral images in the near infrared (NIR) region. Twenty seven of fresh beef samples were collected, spectral data were extracted and the sensory quality attributes were measured. The spectral signatures of beef samples and their corresponding sensory features were then analysed using partial least squares (PLS) regression yielding determination coefficients $\left(R_{c}^{2}\right)$ of $0.97,0.92,0.49$ and 0.93 for predicting juiciness, tenderness, flavour and overall acceptability, respectively. The results are very encouraging and implied that the proposed method has a great potential in nonruinous determination of several sensory attributes simultaneously without tedious sensory panel evaluation. The study recommends applying this non-destructive technique in industrial scales to guarantee high quality of beef and meat products before being marketed to the consumers.
\end{abstract}

Keywords: Spectral analysis, multivariate analysis, beef, quality, sensory attributes.

\section{INTRODUCTION}

The need to develop an automatic method for fast assessment of food quality and composition has fostered intensives research endeavours in using non-destructive techniques based on imaging and spectroscopy methods. For efficient utilizations in food industry, the imaging techniques are able to play a great role for various quality estimation and authentication tasks. Installing computer-integrated imaging techniques helps in evaluating food units in individual basis or as a lot and provides relevant information about their characteristics and quality features. More recently, the applicability of imaging techniques is increasing in different agricultural areas due to the advancement occurred in modern computers and the availability of competent imaging devices (Vote et al., 2003; Shackelford et al., 2005; Calvini et al., 2020).

The traditional imaging technique is able to provide information about the geometry, dimensions, size, appearance and colour of food items. However, this spatial information may be not enough to make some complex classification, detection and/or detection operations. Thus, spectroscopy in different regions of the electromagnetic spectrum has been introduced to give more insight about the internal features of food being examined in term of physical structures and chemical composition (Morsy and Sun, 2013). However, the use of conventional spectroscopic measurement is more suitable for liquids or for homogenous food materials depending only on point scanning measurements. Thus, the spectral imaging technique has been invented to integrate both imaging and spectroscopy in one technique to provide not only spatial information but also spectra details and to visualize properties that either system cannot perform making it suitable for non-homogenous solid foods (Talens et al., 2013).

A relatively large number of research works have been conducted on using spectral imaging techniques for quality evaluation of food and agricultural products, especially fruits, vegetables, grains, meat, poultry and fish (Cluff et al., 2008; Naganathan et al., 2008; Tao et al., 2012; Li et al., 2018; Baek et al., 2019; Oliveri et al., 2019). Therefore, different system configurations have been introduced to measure various quality traits such as water holding capacity, chemical composition, colour, instrumental tenderness in various meat products such as beef (Naganathan et al., 2008; ElMasry et al., 2011), lamb (Kamruzzaman et al., 2013), and poultry (Yoon et al., 2010). The system has been also used successfully in determining meat safety in terms of microbial invasion and detecting the level of contamination (Barbin et al., 2013; Feng et al., 2013; Ye et al., 2016). Unfortunately, there are very limited research endeavours have focused works towards studying the potential applications of spectral imaging techniques in predicting the beef palatability to estimate sensory features of beef.

As the acceptability of meat is usually linked to several sensory features such as tenderness, flavour and juiciness, these features should be determined in relation to the technological features. It is well documented that most of the consumers have no problems to pay more for beef cuts that will meet their eating expectations (Shackelford et al., 2001). In fact, the basic correlation between measurements estimated by various laboratory instruments and those estimated by sensory evaluation by experienced sensory panellists should be deeply considered (Perry et al., 2001; Watson et al., 2008). Experienced panellists are normally trained to score specific attributes of eating quality, but finding a tool to 
objectively determine the sensory features for palatability purposes is more interesting to research. Sensory quality of the muscle longissimus dorsi is of special interest, because this portion of the carcass is more preferable for consumption by the majority of customers (Van Oeckel et al., 1999).

Therefore, the meat industry could benefit from the recent advancement occurred in the expeditious and non-destructive methods to identify and quantify the essential meat sensory parameters without going through complicated process of subjective sensory evaluation. Accordingly, the main aim of this work was to utilize a hyperspectral imaging system employed under the reflectance mode to predict some basic sensory attributes and overall palatability of beef by acquiring spectral images in the near infrared (NIR) region $(910-1700 \mathrm{~nm})$ of the electromagnetic spectrum and build robust PLSR calibration models to relate spectral data and sensory parameters.

\section{MATERIALS AND METHODS}

\section{Samples and image acquisition}

Beef samples $(n=27)$ from $M$. longissimus dorsi (LD) muscle were cut at $24 \mathrm{~h}$ post-mortem from three different breeds at a commercial abattoir and then cut into slices with a thickness of $2.5 \mathrm{~cm}$. The samples were individually scanned using a fully calibrated spectral imaging system. The detailed components of the main configuration of the imaging system used in image acquisition were shown in Figure (1).

The hyperspectral prototype used in this study composed of a camera, a spectrograph, a conveying stage, a computer supported with data acquisition software. The field of view of the camera was illuminated by two $50 \mathrm{~W}$ tungsten-halogen lamps and the conveying stage used to move the sample was driven by a stepping motor with a speed of $0.03 \mathrm{~m} / \mathrm{s}$. The spectral images were collected line by line in a wavelength range of 897-1752 nm with a spectral increment of about 3.34 $\mathrm{nm}$ yielding 256 bands. In a spectral image, the same beef sample is sequentially imaged at each single wavelength interval from 897-1752 $\mathrm{nm}$ yielding 3D hyperspectral image at the end of the imaging process. Due to low signal-to-noise ratio at both ends of the spectrum (between 897-910 nm and 1700-1752 nm), these two regions were removed from the image yielding images in the spectral range of $910 \mathrm{~nm}$ to 1700 nm with 237 bands.

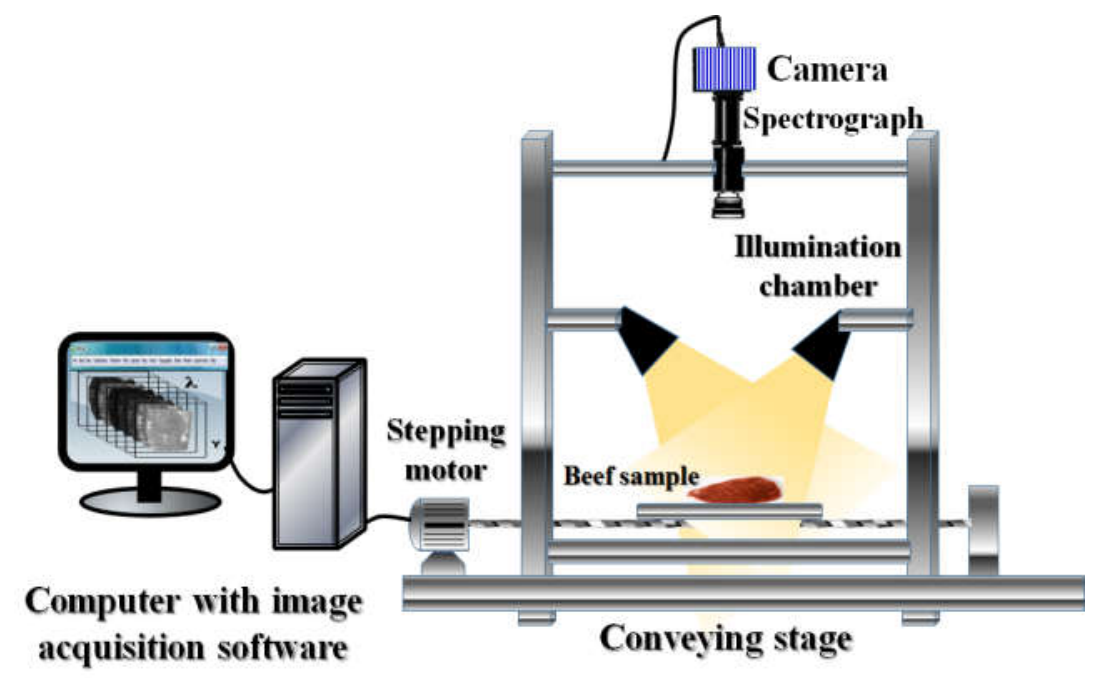

Figure (1): Configuration of the lab-based spectral imaging system used in acquiring spectral images in the NIR spectral range of $910-1700 \mathrm{~nm}$

The acquired spectral image is a threedimensional image called 'hypercube' having two spatial dimensions $(x, y)$ and one spectral dimension $(\lambda)$. To overcome the problem of spectral non-uniformity of the illumination, all acquired images were corrected using two additional images: one is called a dark image ( $0 \%$ reflectance) taken when the light lamps were switched off, and the second one is called a white image taken for a white reference object made from Teflon with reflectance values of $99.9 \%$ with the aid of the following formula:

$$
R(\lambda)=\frac{R_{0}(\lambda)-D(\lambda)}{W(\lambda)-D(\lambda)}
$$

where $R(\lambda), R_{0}(\lambda), D(\lambda)$ and $W(\lambda)$ are the corrected, original, dark and white images at wavelength $\lambda$, respectively.

\section{Sensory evaluation}

The sensory evaluation of beef samples requires panels of human assessors to test and record their responses about the sensory features of the tested beef samples. Directly after image acquisition, beef samples were grilled for $5 \mathrm{~min}$ to a core temperature of $72^{\circ} \mathrm{C}$, divided into equal cubes and randomly served for sensory panel to estimate the beef sensory characteristics in terms of tenderness, juiciness, flavour and overall acceptability. The trained sensory panellists evaluate the sensory attributes inside 
separated booths under red lighting using scale from 1 to 10 for each sensory parameter. Each member of the sensory panel was asked to cleanse his/her mouth between samples by eating a piece of cracker and drinking a sip of water.

\section{Extraction of spectral data}

All steps involved in processing the spectral images, extracting spectral data and building regression models were presented in the schematic diagram shown in Figure (2). As the sensory evaluation was carried out in the whole beef sample, it was important to highlight the sample in every image as the main region of interest (ROI). Selection of the ROIs from the image is a very critical step because it subsequently affects the performance of the developed models.

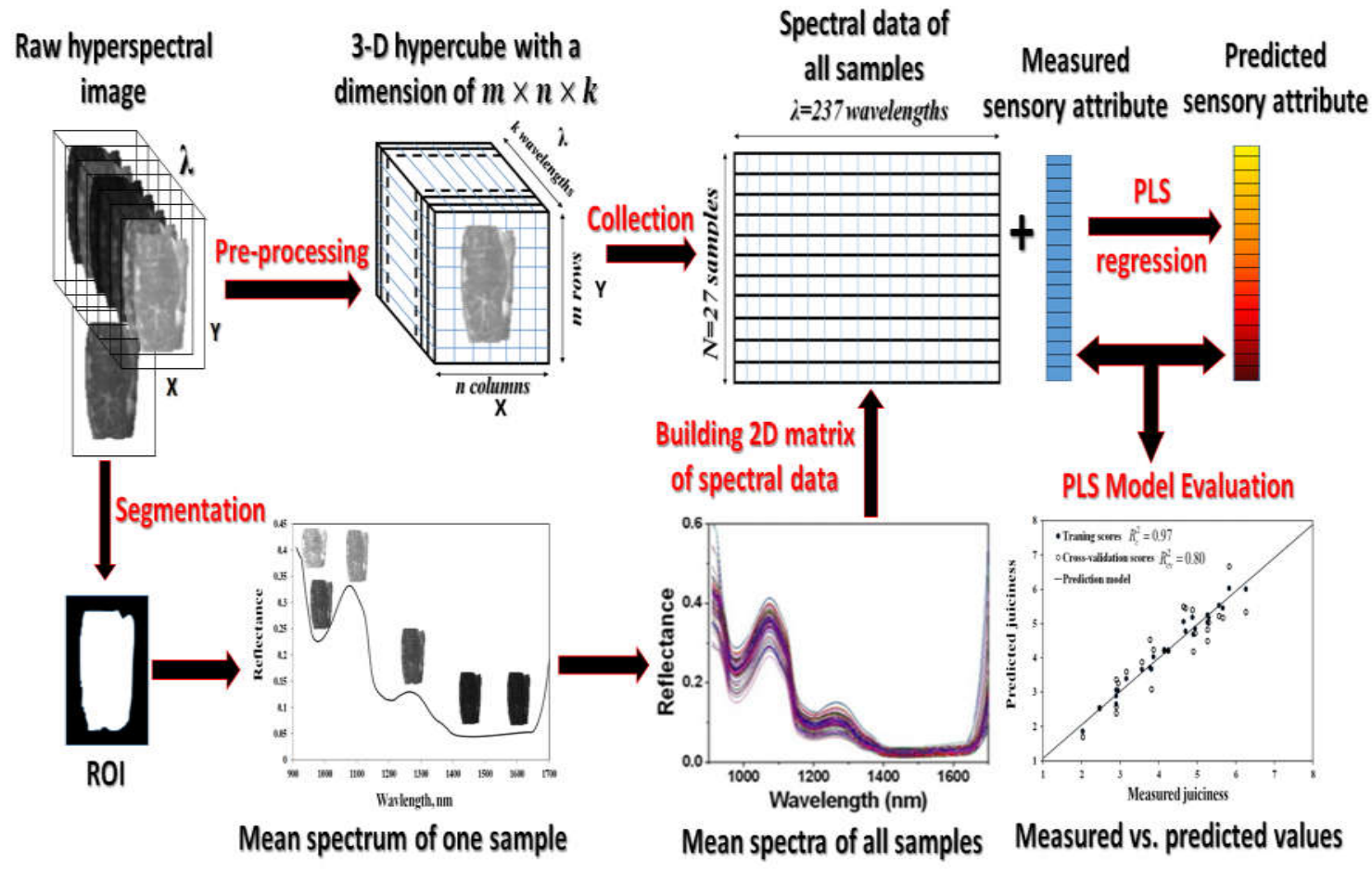

Figure (2): Simplified scheme of the processing steps and analyses of spectral images for predicting sensory parameters using PLS regression. Any spectral image could be spatially displayed at any wavelength as a separate 2D grey image and the spectrum of any pixel in the image could be also viewed as a $2 \mathrm{D}$ plot

The first step in extracting spectral data was to identify all pixels that belong to the beef sample only and discard all other pixels in a process called segmentation. Therefore, one band from the whole hypercube was selected at which a great contrast between the beef sample the background was clear. The segmentation process was carried out on this band to isolate the beef sample from the background as well as from other undesirable pixels which exhibit abnormal reflectance values. The identified part from the segmentation step is called region of interest (ROI) which was then used as a mask to extract spectral data from all pixels corresponding to the beef sample only.

\section{Spectral analysis}

Similar to traditional spectroscopic techniques, spectral imaging method is an indirect technique that entails reference values of the attribute of interest to build calibration models (Sonka et al., 2014; Pathmanaban et al., 2019). To facilitate working in the data, the complex 3-diemnsional hypercube was converted to simple 2-dimentional matrixes in which all pixels belongs to the ROI in the image could be easily accessed. Then, all extracted spectral data from all images in the spectral range of 910-1700 nm were arranged in a matrix (X) having 27 rows (the total number of samples) and 237 columns (the total number of wavelengths in the corrected image). The reference scores of each sensory parameter were saved in a vector (Y) to be modelled with the matrix (X) using partial least squares (PLS) regression. The whole procedure of this analysis is shown in Figure (2).

In the current study, cross-validation using the leave-one-out method was used for validating the developed PLS models for each sensory parameter. In leave-one-out method of cross validation, one sample was kept out and the model was developed in the rest of the samples that then used to predict the sensory value of this sample. This routine was repeated until every sample was kept out once and the overall performance of the model was estimated based on the determination coefficient of the training dataset $\left(R_{c}^{2}\right)$ and those under cross-validation condition $\left(R_{c v}^{2}\right)$. The developed models were also evaluated in terms of the number of latent variables (LV) used in prediction, the standard error in training (SEC) and that of cross validation (SECV). The 
robust model should have high coefficients of determination, less error and least number of latent variables (LV). Moreover, prediction results using raw spectra were compared with those resulting from the estimated sensory scores graphically to evaluate the performance of the model in prediction. Image analysis, segmentation and extraction of spectral data from spectral images were performed using ENVI 4.6 software (ITT, Boulder, CO, USA) and the data treatment and analysis was carried out using Unscrambler 7.9 software (CAMO, Trondheim, Norway).

\section{RESULTS AND DISCUSSIONS \\ Spectral features of beef samples}

As outlined in the previous sections, every corrected spectral image consisted of a series of 237 grey images in the spectral range from 910 to $1700 \mathrm{~nm}$. The average spectrum of the main region of interest (ROI) in the image presents the spectral fingerprint of the whole sample that could be used to characterize the morphological, physical, and chemical characteristics of the beef sample. As shown in Figure (3), spectral signatures of three different beef samples are completely different in their reflectance magnitude indicating that they are basically different in their intrinsic physicochemical properties. Accordingly, the change occurred in these properties is absolutely responsible for the change occurred in their sensory parameters. For instance, samples with high fat content will exhibit low tenderness values but high magnitudes of reflectance. The relationship between spectral profiles shown in Figure (3) and their corresponding reflectance images at different wavelengths shown in Figure (2) is very clear to recognize meaning that a beef sample that looks darker at one wavelength will exhibit low reflectance magnitude and the same sample with high reflectance magnitude will look brighter at this wavelength. For example, the sample at high reflectance values in the spectral profile (e.g. at $950 \mathrm{~nm}$ ) looked brighter than the sample at lower reflectance value in the spectral profile (e.g. at $1450 \mathrm{~nm}$ or $1600 \mathrm{~nm}$ ) which looks very opaque compared to the other wavebands in the spectral profile (Figure 2).

In general, the difference in the spectral fingerprints of the samples in the spectral range of 910-1700 nm was due to difference in scattering properties and physicochemical properties of the examined samples. The reflectance fingerprints of the three different samples shown in Figure (3) exhibited similar patterns (same peaks and valleys) over the entire wavelength region (910-1700 nm). However, the three samples were different in the magnitudes of reflectance especially in the spectral range of 910$1100 \mathrm{~nm}$ and the range of 1650-1700 nm. This could be elucidated to the difference among samples in their physicochemical properties resulting from difference in scattering profiles among these samples. The absorption peaks appeared in this spectral region are mostly related to overtones and combinations of the vibrations of the functional groups that have a hydrogen atom attached to oxygen, carbon or nitrogen such as $\mathrm{C}-\mathrm{H}, \mathrm{N}-\mathrm{H}, \mathrm{O}-\mathrm{H}$ and $\mathrm{S}-\mathrm{H}$ (Cen and $\mathrm{He}$, 2009). Accordingly, the absorption peaks observed at $970 \mathrm{~nm}$ (O-H stretching second overtone) and 1440 $\mathrm{nm}(\mathrm{O}-\mathrm{H}$ stretching first overtones) are related to the presence of water in the beef sample. Meanwhile, the absorption peak observed at $1215 \mathrm{~nm}(\mathrm{C}-\mathrm{H}$ stretching second overtone) is ascribed to fat content in the sample.

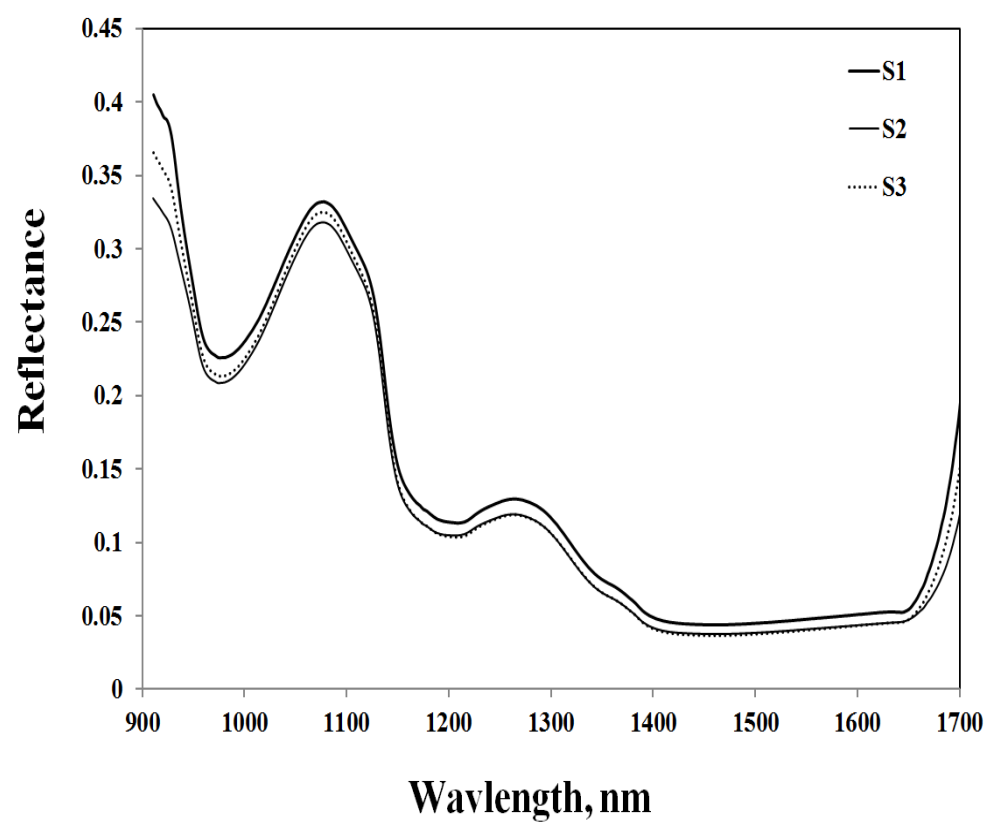

Figure (3): Spectral features of three different beef samples having different tenderness scores (S1: Tender, S2: Intermediate and S3: Tough) extracted from their spectral images in the spectral range of 910-1700 nm 


\section{Sensory attributes}

Sensory traits are very complex characteristics that naturally influenced by so many factors during processing, production and/or handling chains. Also, the difference occurred in these traits are basically due to multiple biochemical factors. In addition to colour, the predominant intrinsic cues for consumers that determine their acceptability of beef are tenderness, juiciness, and flavour (Brewer and Novakofski, 2008). In general, tenderness as the most desirable meat trait is used to measure how easily the meat is chewed or cut and its value depends mostly on the amount of connective tissue and marbling in the meat sample. The juiciness is used to measure the amount of liquids released from the meat after being bitten. On the other hand, the flavour of a piece of meat is a measure of releasing volatile and non-volatile compounds from the lean and fatty tissues after being thermally treated or cooked since uncooked meat has little or no aroma and only a blood-like taste. Thus, the beef eating quality depends on these three factors (i.e. tenderness, juiciness, and flavour) as well as the interrelationships among these attributes. Therefore, the overall acceptability is very important to estimate the consumer satisfaction of beef. In this study, the members of sensory evaluation panel were asked to evaluate each beef sample in terms of juiciness, tenderness, flavour and the overall acceptability. The recorded scores of those sensory attributes of beef samples are presented in Table (1). The panellists recognized a wide range of sensory parameters indicating that there were huge variations among samples.

Considering the subjectivity in the estimation by the sensory panel, the score of each sensory attributes has a wide range of variability among the examined beef samples. In practical terms, the beef samples examined in this study exhibited a wide range of variation in their quality attributes that indicate a good possibility for building robust prediction models. The examined beef samples had a broad range of tenderness (1.65-7.13), juiciness (2.03-6.27), flavour (4.68-7.22) and overall acceptability (1.7-6.78) to develop good calibration models. As declared in Table (1), the average scores of tenderness juiciness, flavour and overall acceptability were $4.71,4.16,5.93$ and 4.75 , respectively.

Table (1): Sensory attributes of beef samples assessed by sensory evaluation panel

\begin{tabular}{lccc}
\hline Quality attribute & Min & Max & Mean \pm SD* \\
\hline Tenderness & 1.65 & 7.13 & $4.71 \pm 1.33$ \\
Juiciness & 2.03 & 6.27 & $5.16 \pm 1.13$ \\
Flavour & 4.68 & 7.22 & $5.93 \pm 0.69$ \\
Overall Acceptability & 1.70 & 6.78 & $4.75 \pm 1.18$ \\
\hline
\end{tabular}

* Values are expressed as the mean scores of the sensory attributes and their corresponding standard deviation (SD)

Prediction of sensory attributes

To accomplish the objectives of this study, the raw spectral data $(\mathrm{X})$ from all beef samples and their corresponding sensory parameter $(\mathrm{Y})$ were compiled as a single dataset. For developing a model for predicting each sensory attribute, a multivariate regression analysis using partial least squares (PLS) regression was used. The performance of predicting the four sensory attributes of beef samples by using PLS regression models was tabulated in Table (2). The presented results revealed that tenderness, juiciness and overall acceptability for the tested beef samples were appropriately predicted with reasonable accuracy. However, the great number of latent factors used to build such prediction models affects their robustness. The poorest model was obtained when predicting flavour since it is a very subjective sensory parameter that differs from person to person. Many studies have been performed to identify which of these sensory traits contributes the most in overall eating satisfaction by the consumers. These results revealed from these studies identified tenderness as the most important sensory trait (Egan et al., 2001). Because tenderness is the most influencing factor that affects the consumer perception of meat eating quality and its palatability category, it was predicted with $\mathrm{R}^{2}$ of 0.92 and 0.74 in the training and cross-validation conditions, respectively. Resembling to tenderness, the juiciness and overall acceptability were predicted with determination coefficient of $\mathrm{R}^{2} 0.80$ and 0.75 with standard error of SEC of 0.18 and 0.31 , respectively. As a try to improve these prediction models, different preprocessing treatments were also experienced on the raw spectral data but they did not improve the performance of the prediction models of all sensory attributes. 
Table (2): Performance of PLSR models in the prediction of various sensory attributes*

\begin{tabular}{|c|c|c|c|c|c|}
\hline Sensory Parameter & $\mathbf{L V}$ & $R_{c}^{2}$ & $R_{c v}^{2}$ & SEC & SECV \\
\hline Tenderness & 10 & 0.92 & 0.74 & 0.37 & 0.69 \\
\hline Juiciness & 12 & 0.97 & 0.80 & 0.18 & 0.54 \\
\hline Flavour & 2 & 0.49 & 0.32 & 0.43 & 0.52 \\
\hline Overall acceptability & 9 & 0.93 & 0.75 & 0.31 & 0.62 \\
\hline
\end{tabular}

* LV: latent variables, $R_{c}^{2}$ : Determination coefficient of calibration, $R_{c v}^{2}$ : Determination coefficient in cross validation, SEC: standard error of calibration and SECV: standard error of cross validation.

To visualize the prediction power of the developed PLS models for each sensory parameter, the measured sensory scores estimated by the sensory panel were graphically illustrated against those predicted by the PLS models. As illustrated in Figure (4), a quite linear trend was noticed in predicting the sensory parameters non-destructively using the spectral features of the analysed beef samples. The lower prediction coefficient for flavour attribute was conceivably due to the narrow range of this parameter of the testes beef samples (Table 1).
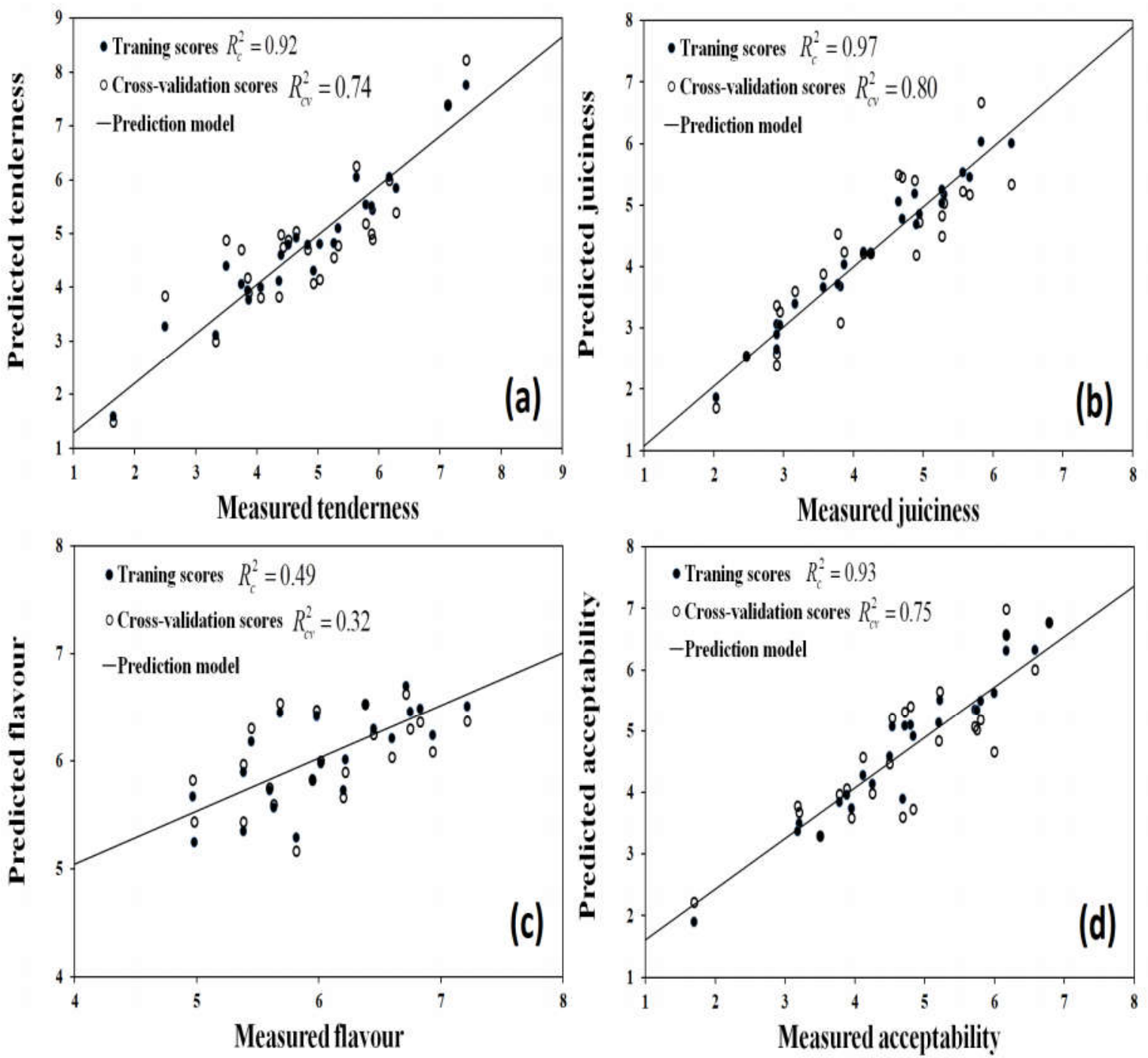

Figure (4): Measured vs. predicted scores of (a) tenderness, (b) juiciness, (c) flavour and (d) overall acceptability of beef samples using PLS regression method 
Because it is not necessarily important to predict the exact values of the sensory attributes, the samples could be classified to different categories based on the values of these attributes. For instance, samples could be classified into three categories (tender, intermediate and tough) and a classification model could be developed instead of a regression model under this categorization. Accordingly, the prediction of these sensory attributes could be improved if the samples are segregated into different categories rather than predicting the exact value of sensory traits. On the other hand, using a small beef data set negatively affects the accuracy of prediction. Therefore, developing more robust models could be achieved by increasing the number of beef samples during building such models.

\section{CONCLUSION}

A hyperspectral imaging technique employed in the reflectance mode in the near infrared (NIR) region was utilized for a non-ruinous determination of beef sensory attributes. The results are promising and revealed that the system and the associated analytical method proposed in this study had a good potential for non-invasive prediction of sensory parameters of beef samples. Because the traditional methods for determining palatability are highly destructive and timeconsuming, the proposed method using the suggested protocol of hyperspectral imaging system was applied for simultaneous estimation of chemical constitutes and palatability. This non-destructive scenario is very beneficial for food science and technology to save time, labour and cost. The method provides simultaneous exploration of information from both spectral and spatial data, allowing the analysis of invisible profiles. The current study recommends applying this nondestructive technique in industrial scales to guarantee high quality of beef and meat products before being marketed to the consumers. The technique can be installed in the beef inspection facilities as a key component of a computer-integrated system to guarantee consumer satisfaction.

\section{REFERENCES}

Baek, I., B. K. Cho, S. A. Gadsden, C. Eggleton, M. Oh, C. Mo and M. S. Kim (2019). A novel hyperspectral line-scan imaging method for whole surfaces of round shaped agricultural products. Biosystems Engineering, 188: 57-66.

Barbin, D. F., G. ElMasry, D. W. Sun, P. Allen and N. Morsy (2013). Non-destructive assessment of microbial contamination in porcine meat using NIR hyperspectral imaging. Innovative Food Science \& Emerging Technologies, 17: 180191.

Brewer, S. and J. Novakofski (2008). Consumer sensory evaluations of aging effects on beef quality. Journal of Food Science, 73(1): S78-S82.

Calvini, R., S. Michelini, V. Pizzamiglio, G. Foca and A. Ulrici (2020). Exploring the potential of NIR hyperspectral imaging for automated quantification of rind amount in grated
Parmigiano Reggiano cheese. Food Control, 12, Article No. 107111.

Cen, H. and R. Lu (2009). Quantification of the optical properties of two-layer turbid materials using a hyperspectral imaging-based spatially-resolved technique. Applied Optics, 48(29): 5612-5623.

Cluff, K., G. K. Naganathan, J. Subbiah, R. LU, C. R. Calkins and A. Samal (2008). Optical scattering in beef steak to predict tenderness using hyperspectral imaging in the VIS-NIR region. Sensing and Instrumentation Food Quality, 2: 189-196.

Egan, A. F., D. M. Ferguson and J. M. Thompson (2001). Consumer sensory requirements for beef and their implications for the Australian beef industry. Australian Journal of Experimental Agriculture, 41(7): 855-859.

ElMasry, G., D. W. Ssun and P. Allen (2011). Nondestructive determination of water-holding capacity in fresh beef by using NIR hyperspectral imaging. Food Research International, 44: 2624-2633

Feng, Y. Z., G. ElMasry, D. W. Sun, A. G. Scannell, D. Walsh and N. Morcy (2013). Near-infrared hyperspectral imaging and partial least squares regression for rapid and reagentless determination of Enterobacteriaceae on chicken fillets. Food Chemistry, 138(2-3): 1829-1836.

Kamruzzaman, M., G. ElMasry, D. W. Sun and P. Allen (2013). Non-destructive assessment of instrumental and sensory tenderness of lamb meat using NIR hyperspectral imaging. Food Chemistry, 141(1): 389-396.

Li, B., M. Cobo-Medina, J. Lecourt, N. Harrison, R. J. Harrison and J. V. Cross (2018). Application of hyperspectral imaging for nondestructive measurement of plum quality attributes. Postharvest Biology and Technology, 141: 815.

Morsy, N. and D. W. Sun (2013). Robust linear and non-linear models of NIR spectroscopy for detection and quantification of adulterants in fresh and frozen-thawed minced beef. Meat science, 93(2): 292-302.

Naganathan, G. K., L. M. Grimes, J. Subbiah, C. R. Calkins, A. Samal and G. E. Meyer (2008).Visible/near-infrared hyperspectral imaging for beef tenderness prediction. Computers and Electronics in Agriculture, 64(2): 225-233.

Oliveri, P., C. Malegori, M. Casale, E. Tartacca and G. Salvatori (2019). An innovative multivariate strategy for HSI-NIR images to automatically detect defects in green coffee. Talanta, 199: 270-276.

Pathmanaban, P., B. K. Gnanavel and S. S. Anandan (2019). Recent Application of imaging techniques for Fruit quality Assessment. Trends in Food Science \& Technology, 94(1): 32-42.

Perry, D., J. M. Thompson, I. H. Hwang, A. Butchers and A. F. Egan (2001). Relationship between 
objective measurements and taste panel assessment of beef quality. Australian Journal of Experimental Agriculture, 41: 981-989.

Shackelford, S. D., T. L. Wheeler, M. K. Meade, J. O. Reagan, B. L. Byrnes and M. Koohmaraie (2001). Consumer impressions of Tender Select beef. Journal of Animal Science, 79(10): 2605-2614.

Shackelford, S. D., T. L. Wheeler and M. Koohmaraie (2005). On-line classification of US Select beef carcasses for longissimus tenderness using visible and near-infrared reflectance spectroscopy. Meat Science, 69(3): 409-415.

Sonka, M., V. Hlavac and R. Boyle (2014). Image processing, analysis, and machine vision: Cengage Learning, Stamford, USA.

Talens, P., L. Mora, N. Morsy, D. F. Barbin, G. ElMasry and D. W. Sun (2013). Prediction of water and protein contents and quality classification of Spanish cooked ham using NIR hyperspectral imaging. Journal of Food Engineering, 117(3): 272-280.

Tao, F., Y. Peng, Y. Li, K. Chao and S. Dhakal (2012). Simultaneous determination of tenderness and Escherichia coli contamination of pork using hyperspectral scattering technique. Meat Science, 90: 851-857.

VanOeckel, M. J., N. Warnants and C. V. Boucque (1999). Pork tenderness estimation by taste panel, Warner-Bratzlershear force and on-line methods. Meat Science, 53: 259-267.

Vote, D. J., K. E. Belk, J. D. Tatum, J. A. Scanga and G. C. Smith (2003). Online prediction of beef tenderness using a computer vision system equipped with a Beef Cam module. Journal of Animal Science, 81: 457-465.

Watson, R., A. Gee, R. Polkinghorne and M. Porter (2008). Consumer assessment of eating quality - development of protocols for Meat Standards Australia (MSA) testing. Australian Journal of Experimental Agriculture, 48: 1360-1367.

Ye, X., K. Iino and S. Zhang (2016). Monitoring of bacterial contamination on chicken meat surface using a novel narrowband spectral index derived from hyperspectral imagery data. Meat science, 122: 25-31.

Yoon, S. C., K. C. Lawrence, J. E. Line, G. R. Siragusa, P. W. Feldner and B. Park (2010). Detection of Campylobacter colonies using hyperspectral imaging. Sensing and Instrumentation for Food Quality and Safety, 4(1): 35-49.

\section{التتبؤ بالصفات الحسية للحم البقرى الطازج باستخدام التحليل الطيفى المثقم

$$
\text { قسم علوم وتكنولوجيا الأغذية (فرع الاقتصاد المنزلي)، كلية الزر اعة، جامعة قناة السويس، الإسماعلية }
$$

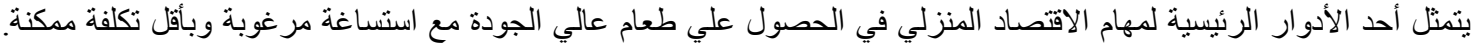

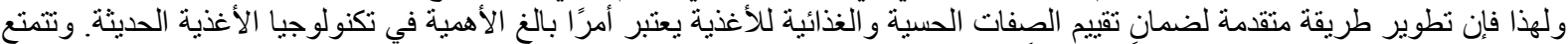

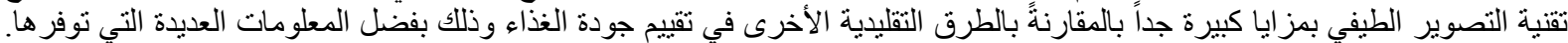

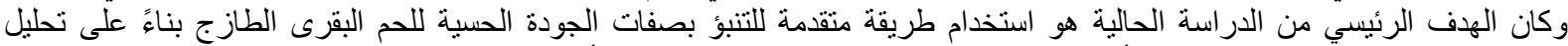

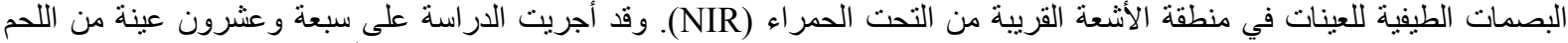

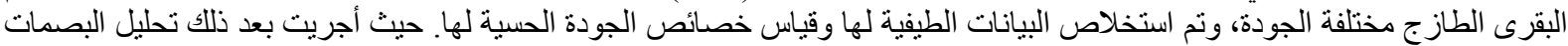

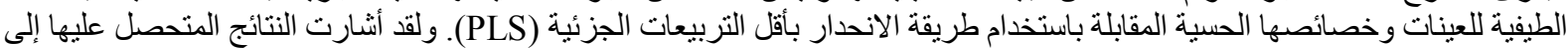

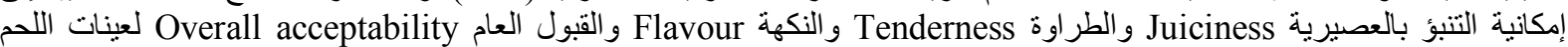

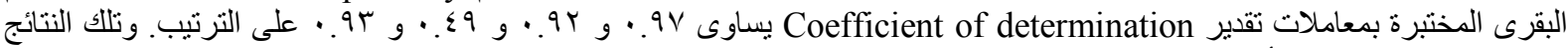

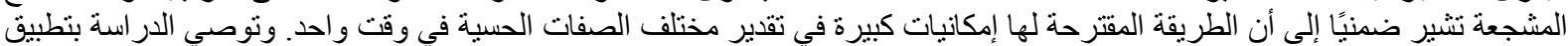

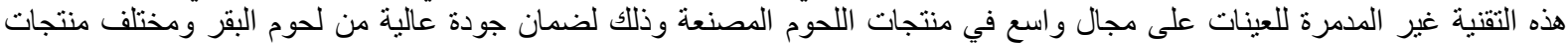

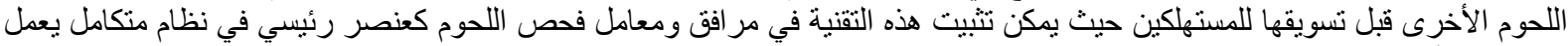

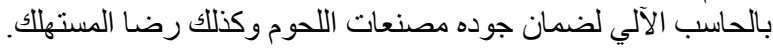

الكلمات المفتاحية: القياس الكمي ـ التحليل الطيفى المتقدم ـ لحم البقر ـ الصفات الحسية 